\title{
Unsteady MHD flow of a visco-elastic fluid along vertical porous surface with fluctuating temperature and concentration
}

\author{
Arpita Mohanty, Pravat Kumar Rath, G. C. Dash \\ ${ }^{l}$ Department of Mathematics, Prananath Autonomous College, Khordha-752057, Odisha, India \\ ${ }^{2}$ Dept. of Mathematics, B.R.M. International Institute of Technology, Bhubaneswar-751010, Odisha, India \\ ${ }^{3}$ Department of Mathematics, S.O.A. University, Bhubaneswar-751030, Odisha, India
}

\begin{abstract}
An unsteady magneto-hydrodynamics flow of an incompressible visco-elastic fluid [Walters' fluid (Model $\left.\mathrm{B}^{\prime}\right)$ ] along an infinite hot vertical porous surface with fluctuating temperature and concentration has been studied. The governing equations of motion, energy and concentration are solved by the successive perturbation techniques. The expressions for the skin friction, Nusselt number and Sherwood number are also derived. The variations in the fluid velocity, temperature and concentration are shown graphically whereas numerical values of skin friction, Nusselt number and Sherwood number are presented in a tabular form for various values of pertinent flow parameters.
\end{abstract}

Keywords: - MHD flow; porous medium; chemical reaction/heat source

\section{INTRODUCTION}

Flow between porous boundaries is of practical, as well as theoretical interest. The practical interest includes problems of gaseous diffusion, transpiration cooling, lubrication of porous bearing etc. Flow through porous medium under the influence of temperature and concentration differences find great applications in Geothermy, Geophysics and Technology.

Several researches [1-6] have studied the two dimensional free convection MHD flow with heat and mass transfer. Walters [7] have analyzed second order effects in elasticity plasticity and fluid dynamics. Sharma and Pareek [8] later examined the unsteady flow and heat transfer through an elastico-viscous liquid along an infinite hot vertical porous moving plate with variable free stream and suction. Flow and heat transfer of an electrically conducting visco-elastic fluid between two horizontal squeezing/stretching plates have been studied by Rath et al.[9].

Alhabi et al.[10] have analyzed heat and mass transfer in MHD visco-elastic fluid flow through a porous medium over a stretching sheet with chemical reaction. Kumar and Sivraj [11] have studied MHD mixed convective visco-elastic fluid flow in a permeable vertical channel with Dufour effect and chemical reaction parameter. Damseh and Sharnaik [12] have also studied visco-elastic fluid flow past an infinite vertical porous plate in the presence of first order chemical reaction. MHD flow through a porous medium past a stretched vertical permeable surface in the presence of heat source/sink and a chemical reaction has been studied by Dash et al.[13]. Rath et al. [14] have studied the three dimensional free convection flow through porous medium in a vertical channel with heat source and chemical reaction. Sharma and Sharma [15] have studied the unsteady two dimensional flow and heat transfer through an elastico-viscous liquid along an infinite hot vertical porous surface bounded by porous medium.

The main objective of the present study is to investigate the combined effect of magnetic field, heat source and chemical reaction on two-dimensional visco-elastic flow through porous media in a vertical surface with fluctuating temperature and concentration. The governing equations of flow under the Boussinesq approximation are solved analytically using successive perturbation method.

\section{FORMULATION OF THE PROBLEM}

An unsteady two dimensional MHD flow past a vertical infinite surface embedded in a porous medium has been considered with the visco-elastic fluid model (Walters' fluid (Model B') [7]). A uniform transverse magnetic field $\mathrm{B}_{0}$ is applied normal to the direction of the fluid flow. The $\mathrm{x}^{*}$-axis is taken along the surface in upwards direction i.e., opposite to the direction of gravity and $\mathrm{y}^{*}$-axis is taken normal to the surface. The governing equations of continuity, motion, energy and species concentration for flow of a visco-elastic fluid through porous medium bounded by an infinite, vertical porous surface with oscillatory suction velocity are given by:

$$
\frac{\partial v^{*}}{\partial y^{*}}=0
$$




$$
\begin{aligned}
& \left(\frac{\partial u^{*}}{\partial t^{*}}+v^{*} \frac{\partial u^{*}}{\partial y^{*}}\right)=-\frac{1}{\rho} \frac{\partial p}{\partial x^{*}}-g+v \frac{\partial^{2} u^{*}}{\partial y^{* 2}}-\frac{v}{k^{*}} u^{*} \\
& -\frac{K_{0}^{*}}{\rho}\left(\frac{\partial^{3} u^{*}}{\partial t^{*} \partial y^{* 2}}+v^{*} \frac{\partial^{3} u^{*}}{\partial y^{* 3}}\right)-\frac{\sigma B_{0}^{2} u^{*}}{\rho} \\
& \frac{\partial T^{*}}{\partial t^{*}}+v^{*} \frac{\partial T^{*}}{\partial y^{*}}=\frac{k}{\rho C_{p}} \frac{\partial T^{* 2}}{\partial y^{* 2}}+S^{*}\left(T^{*}-T_{\infty}\right) \\
& \frac{\partial C^{*}}{\partial t^{*}}+v^{*} \frac{\partial C^{*}}{\partial y^{*}}=D \frac{\partial^{2} C^{*}}{\partial y^{* 2}}-K_{c}^{*}\left(C^{*}-C_{\infty}\right)
\end{aligned}
$$

Where $\mathrm{u}^{*}, \mathrm{v}^{*}$ denote the components of velocity along $\mathrm{x}^{*}$ and $\mathrm{y}^{*}$ directions respectively, $\rho$ the density of the fluid, $\mathrm{g}$ the acceleration due to gravity, $v$ the Kinematic viscosity, $k^{*}$ the permeability parameter $K_{0}^{*}$ the non-Newtonian parameter, $\mathrm{T}^{*}$ the temperature of the fluid, $\mathrm{k}$ the thermal conductivity of fluid, $\mathrm{C}_{\mathrm{p}}$ the specific heat of the fluid at constant pressure, $\mathrm{t}^{*}$ the time, $\mathrm{C}^{*}$ the concentration of fluid, $K_{c}^{*}$ the chemical reaction parameter, D the mass diffusion coefficient and $\sigma$ the electrical conductivity.

The boundary conditions are given by:

$$
\begin{aligned}
& \mathrm{t}>0: y^{*}=0 ; u^{*}=0, T^{*}=T_{w}+\varepsilon\left(T_{w}-T_{\infty}\right) e^{i \omega t}, C^{*}=C_{w}+\varepsilon\left(C_{w}-C_{\infty}\right) e^{i \omega t} \\
& y^{*} \rightarrow \infty ; u^{*} \rightarrow U^{*}\left(t^{*}\right), T^{*} \rightarrow T_{\infty}, C^{*} \rightarrow C_{\infty}
\end{aligned}
$$

where $T_{w}$ and $\mathrm{C}_{\mathrm{w}}$ are the surface temperature and concentration respectively, $\mathrm{U}^{*}\left(\mathrm{t}^{*}\right)$ the free stream velocity, $\mathrm{T}_{\infty}$ the free stream temperature and $\mathrm{C}_{\infty}$ is the concentration of the fluid for away from the wall.

In view of Bousinesq approximation, the equation (2) is reduced to

$$
\begin{aligned}
& \rho\left(\frac{\partial u^{*}}{\partial t^{*}}+v^{*} \frac{\partial u^{*}}{\partial y^{*}}\right)=\rho \frac{\partial U^{*}}{\partial t^{*}}+\frac{\mu}{k^{*}}\left(U^{*}-u^{*}\right)+\mu \frac{\partial^{2} u^{*}}{\partial y^{* 2}} \\
& -K_{0}^{*}\left(\frac{\partial^{3} u^{*}}{\partial t^{*} \partial y^{* 2}}+v^{*} \frac{\partial^{3} u^{*}}{\partial y^{* 3}}\right)+\sigma B_{0}^{2}\left(U^{*}-u^{*}\right)+g \beta \rho\left(T^{*}-T_{\infty}\right) \\
& +g \beta^{*} \rho\left(C^{*}-C_{\infty}\right)
\end{aligned}
$$

where $\beta$ and $\beta^{*}$ are the volumetric coefficient of thermal and concentration expansion respectively and $\mu$ the coefficient of dynamic viscosity.

From equation of community (1), it is clear that the suction velocity normal to the plate is either a constant or a function of time. Here it is assumed in the form of

$$
\mathrm{v}^{*}=-\mathrm{v}_{0}\left(1+\varepsilon \mathrm{e}^{\mathrm{i} \omega^{* *} \mathrm{t}}\right)
$$

where, $\omega^{*}$ the frequency of vibration, $\varepsilon$ is a small parameter i.e., $0<\varepsilon<1$ and $\mathrm{v}_{0}$ is a non-zero positive suction velocity. Here the negative sign indicates that the suction is towards the plate.

The governing equations of the flow are non-dimentionalised using the following transformation.

$$
\begin{gathered}
y=\frac{y^{*} v_{0}}{v}, t=\frac{t^{*} v_{0}^{2}}{4 v}, u=\frac{u^{*}}{U}, \omega=\frac{4 v \omega^{*}}{v_{0}^{2}}, \\
U(t)=\frac{U^{*}\left(t^{*}\right)}{U}, T=\frac{T^{*}-T_{\infty}}{T_{w}-T_{\infty}}, P_{r}=\frac{\mu C_{p}}{k} \\
\mathrm{k}_{\mathrm{p}}=\frac{k^{*} v_{0}^{2}}{v^{2}}, K_{0}=\frac{K_{0}^{*} v_{0}^{2}}{\rho v^{2}}, G_{r}=\frac{v g \beta\left(T_{w}-T_{\infty}\right)}{U v_{0}^{2}}, \\
\mathrm{M}=\frac{\sigma \mathrm{B}_{0}^{2} v}{\rho v_{0}^{2}}, K_{c}=\frac{K_{c}^{*} v}{v_{0}^{2}}
\end{gathered}
$$




$$
\begin{aligned}
& \mathrm{C}= \frac{C^{*}-C_{\infty}}{C_{w}-C_{\infty}}, S=\frac{S^{*} v}{v_{0}^{2}}, G_{c}=\frac{v g \beta^{*}\left(C_{w}-C_{\infty}\right)}{U v_{0}^{2}} \\
& \quad \text { In view of equations (7) and (8), equations (3) - (4) and (6) become : } \\
& \quad \frac{\partial^{2} u}{\partial y^{2}}+\left(1+\varepsilon e^{i \omega t}\right) \frac{\partial u}{\partial y}=\frac{1}{4} \frac{\partial u}{\partial t}-G_{r} T-G_{c} C+\left(\frac{1}{k_{p}}+M\right) u-\left(\frac{1}{k_{p}}+M\right)\left(1+\varepsilon e^{i \omega t}\right) \\
&-\frac{1}{4} \varepsilon i \omega e^{i \omega t}+k_{0}\left[\frac{1}{4} \frac{\partial^{3} u}{\partial t \partial y^{2}}-\left(1+\varepsilon e^{i w t}\right) \frac{\partial^{3} u}{\partial y^{3}}\right] \\
& \frac{P_{r}}{4} \frac{\partial T}{\partial t}=\frac{\partial^{2} T}{\partial y^{2}}+P_{r}\left(1+\varepsilon e^{i \omega t}\right) \frac{\partial T}{\partial y}+P_{r} S T \\
& \frac{S_{c}}{4} \frac{\partial C}{\partial t}=\frac{\partial^{2} C}{\partial y^{2}}+S_{c}\left(1+\varepsilon e^{i \omega t}\right) \frac{\partial C}{\partial y}-S_{c} K_{c} C
\end{aligned}
$$

where $G_{r}$ the Grashof number for heat transfer, $P_{r}$ the Prandtl number, $K_{0}$ the non-Newtonian parameter, $G_{c}$ the Grashof number for mass transfer, $S_{c}$ the Schmidt number, $M$ the magnetic parameter and $k_{p}$ the porosity parameter.

The corresponding boundary conditions in non-dimensional form:

$$
\begin{aligned}
& y=0: u=0, T=1+\varepsilon e^{i \omega t}, C=1+\varepsilon e^{i \omega t} \\
& y \rightarrow \infty: u \rightarrow U(t)=1+\varepsilon e^{i \omega t}, T \rightarrow 0, C \rightarrow 0
\end{aligned}
$$

Solution procedure

$$
\begin{aligned}
& \text { In view of the above assumption it is justified to assume that : } \\
& u(y, t)=u_{0}(y)+\varepsilon u_{1}(y) e^{i \omega t} \\
& \mathrm{~T}(\mathrm{y}, \mathrm{t})=\mathrm{T}_{0}(\mathrm{y})+\varepsilon \mathrm{T}_{1}(\mathrm{y}) \mathrm{e}^{\mathrm{i} \omega t} \\
& \mathrm{C}(\mathrm{y}, \mathrm{t})=\mathrm{C}_{0}(\mathrm{y})+\varepsilon \mathrm{C}_{1}(\mathrm{y}) \mathrm{e}^{\mathrm{i} \omega t}
\end{aligned}
$$

Substituting equations(13) - (15) into equations (9) - (11) and equating the coefficients of $\varepsilon^{0}$ and $\varepsilon$, we get

\section{Zeroth order :}

$$
\begin{aligned}
& K_{0} u_{0}^{\prime \prime \prime}+u_{0}^{\prime \prime}+u_{0}^{\prime}-\left(M+\frac{1}{k_{p}}\right) \mathrm{u}_{0}=-\mathrm{G}_{\mathrm{r}} \mathrm{T}_{0}-\left(M+\frac{1}{k_{p}}\right)-G_{c} C_{0} \\
& T_{0}^{\prime \prime}+P_{r} T_{0}^{\prime}+P_{r} S T_{0}=0 \\
& C_{0}^{\prime \prime}+S_{c} C_{0}^{\prime}-K_{c} S_{c} C_{0}=0
\end{aligned}
$$

First order:

$$
\begin{aligned}
& K_{0} u_{1}^{\prime \prime \prime}+\left(1-\frac{k_{0} i \omega}{4}\right) u_{1}^{\prime \prime}+u_{1}^{\prime}-\left(M+\frac{1}{k_{p}}+\frac{i \omega}{4}\right) u_{1} \\
& \quad=-\mathrm{G}_{\mathrm{r}} \mathrm{T}_{1}-\mathrm{G}_{\mathrm{c}} \mathrm{C}_{1}-\mathrm{K}_{0} u_{0}^{\prime \prime \prime}-u_{0}^{\prime}-\left(M+\frac{1}{k_{p}}+\frac{i \omega}{4}\right) \\
& T_{1}^{\prime \prime}+P_{r} T_{1}^{\prime}+P_{r}\left(S-\frac{i \omega}{4}\right) T_{1}=-P_{r} T_{0}^{\prime} \\
& C_{1}^{\prime \prime}+S_{c} C_{1}^{\prime}-\left(\frac{i \omega}{4}+k_{c}\right) S_{c} C_{1}=-S_{c} C_{0}^{\prime}
\end{aligned}
$$

where prime denote differentiation with respect to $\mathrm{y}$.

The corresponding boundary conditions (12) are reduced to :

$$
\begin{aligned}
& \mathrm{y}=0: \mathrm{u}_{0}=0, \mathrm{u}_{1}=0, \mathrm{~T}_{0}=1, \mathrm{~T}_{1}=1, \mathrm{C}_{0}=1, \mathrm{C}_{1}=1, \\
& \mathrm{y} \rightarrow \infty: u_{0} \rightarrow 1, u_{1} \rightarrow 1, T_{0} \rightarrow 0, T_{1} \rightarrow 0, C_{0} \rightarrow 0, C_{1} \rightarrow 0
\end{aligned}
$$

Solving equations (17), (18), (20) and (21) under the boundary conditions (22), we get 


$$
\begin{aligned}
\mathrm{T}_{0} & =e^{m_{1} y} \\
\mathrm{C}_{0} & =e^{m_{3} y} \\
T_{1} & =\left(1+\frac{4 i}{\omega} m_{1}\right) e^{m_{2} y}-\frac{4 i}{\omega} m_{1} e^{m_{1} y} \\
C_{I} & =\left(1+\frac{4 i}{\omega} m_{3}\right) e^{m_{4} y}-\frac{4 i}{\omega} m_{3} e^{m_{3} y}
\end{aligned}
$$

The equations (16) and (19) are still of third order where $\mathrm{K}_{0} \neq 0$ and reduce to second order differential equation when $\mathrm{K}_{0}=0$ i.e., for a Newtonian fluid case. Hence, the presence of a non-Newtonian parameter increases the order of differential equation. While from physical consideration only two boundary conditions are available. Since the non-Newtonian parameter $\left(\mathrm{K}_{0}\right)$ is very small for incompressible fluid (Walters ${ }^{7}$ ), therefore $\mathrm{u}_{0}$ and $\mathrm{u}_{1}$ can be expanded in powers of $\mathrm{K}_{0}$ which is given by

$$
\begin{aligned}
& u_{0}(y)=u_{00}(y)+K_{0} u_{01}(y)+O\left(K_{0}^{2}\right) \\
& u_{I}(y)=u_{10}(y)+K_{0} u_{11}(y)+O\left(K_{0}^{2}\right)
\end{aligned}
$$

introducing equation (27) into equations (16) and (19), we obtain the following systems of equations.

$$
\begin{aligned}
& u_{00}^{\prime \prime}+u_{00}^{\prime}-\left(M+\frac{1}{k_{p}}\right) u_{00}=-\left(M+\frac{1}{k_{p}}\right)-G_{r} T_{0}-G_{c} C_{0} \\
& u_{10}^{\prime \prime}+u_{10}^{\prime}-\left(M+\frac{1}{k_{p}}+\frac{i \omega}{4}\right) u_{10}=-G_{r} T_{1}-G_{c} C_{1}-u_{00}^{\prime}-\left(M+\frac{1}{k_{p}}+\frac{i \omega}{4}\right) \\
& u_{01}^{\prime \prime}+u_{01}^{\prime}-\left(M+\frac{1}{k_{p}}\right) u_{01}=-u_{00}^{\prime \prime \prime} \\
& u_{11}^{\prime \prime}+u_{11}^{\prime}-\left(M+\frac{1}{k_{p}}+\frac{i \omega}{4}\right) u_{11}=-u_{00}^{\prime \prime \prime}-u_{01}^{\prime}-u_{10}^{\prime \prime \prime}+\frac{i \omega}{4} u_{10}^{\prime \prime}
\end{aligned}
$$

With the corresponding boundary conditions:

$\left.\begin{array}{l}\mathrm{y}=0, \mathrm{u}_{00}=0, \mathrm{u}_{01}=0, \mathrm{u}_{10}=0, \mathrm{u}_{11}=0 \\ \mathrm{y} \rightarrow \infty: u_{00} \rightarrow 1, u_{01} \rightarrow 0, u_{10} \rightarrow 1, u_{11} \rightarrow 0\end{array}\right\}$

Solving equations (28) - (31) under the boundary condition (32), we get

$\mathrm{u}_{00}=\left(\mathrm{A}_{3}+\mathrm{A}_{4}-1\right) e^{m_{5} y}-A_{3} e^{m_{1} y}-A_{4} e^{m_{3} y}+1$

$\mathrm{u}_{01}=\mathrm{A}_{9} e^{m_{5} y}+A_{7} e^{m_{1} y}+A_{8} e^{m_{3} y}$

$\mathrm{u}_{10}=\mathrm{A}_{19} e^{m_{6} y}+A_{17} e^{m_{1} y}+A_{18} e^{m_{3} y}+A_{10} e^{m_{2} y}+A_{14} e^{m_{5} y}+A_{12} e^{m_{4} y}+1$

$\mathrm{u}_{11}=\mathrm{A}_{38} e^{m_{6} y}+A_{35} e^{m_{5} y}+A_{28} e^{m_{4} y}+A_{36} e^{m_{3} y}+A_{26} e^{m_{2} y}+A_{37} e^{m_{1} y}$

Finally, the velocity $\mathrm{u}(\mathrm{y}, \mathrm{t})$ is given by

$$
\begin{aligned}
& \mathrm{u}(\mathrm{y}, \mathrm{t})=\left(\mathrm{A}_{3}+\mathrm{A}_{4}-1\right) e^{m_{5} y}-A_{3} e^{m_{1} y}-A_{4} e^{m_{3} y}+1+K_{0}\left(A_{9} e^{m_{5} y}+A_{7} e^{m_{1} y}+A_{8} e^{m_{3} y}\right) \\
& +\varepsilon\left[\left\{A_{19} e^{m_{6} y}+A_{17} e^{m_{1} y}+A_{18} e^{m_{3} y}+A_{10} e^{m_{2} y}+A_{14} e^{m_{5} y}+A_{12} e^{m_{4} y}+1\right\}\right. \\
& \left.+K_{0}\left\{A_{38} e^{m_{6} y}+A_{35} e^{m_{5} y}+A_{28} e^{m_{4} y}+A_{36} e^{m_{3} y}+A_{26} e^{m_{2} y}+A_{37} e^{m_{1} y}\right\}\right] e^{i \omega t}
\end{aligned}
$$

where $\mathrm{A}_{1}-\mathrm{A}_{38}, \mathrm{~m}_{1}-\mathrm{m}_{6}$ are given in the Appendix.

\section{Skin friction $\left(\mathbf{C}_{\mathrm{f}}\right)$}




$$
C_{f}=\frac{\tau_{w}^{*}}{\rho U_{0} v}=\left[\frac{\partial u}{\partial y}-K_{0}\left(\frac{1}{4} \frac{\partial^{2} u}{\partial t \partial y}-\left(1+\varepsilon e^{i \omega t}\right) \frac{\partial^{2} u}{\partial y^{2}}\right)\right]_{y=0}
$$

Nusselt Number $\left(\mathbf{N}_{\mathbf{u}}\right)$

$$
\begin{gathered}
\mathrm{Nu}=\frac{q v}{v_{0} k\left(T_{w}-T_{\infty}\right)}=\left(\frac{\partial T}{\partial y}\right)_{y=0} \\
\therefore N_{u}=-\left[m_{1}+\varepsilon e^{i \omega t}\left\{\left(1+\frac{4 i}{\omega} m_{1}\right) m_{2}-\frac{4 i}{\omega} m_{1}^{2}\right\}\right]
\end{gathered}
$$

Sherwood Number $\left(\mathbf{S}_{\mathrm{h}}\right)$

$$
\begin{aligned}
& S_{h}=\frac{m v}{v_{0} D\left(C_{w}-C_{\infty}\right)}=-\left(\frac{\partial c}{\partial y}\right)_{y=0} \\
& =-\left[m_{3}+\varepsilon e^{i \omega t}\left\{\left(1+\frac{4 i}{\omega} m_{3}\right) m_{4}-\frac{4 i}{\omega} m_{3}^{2}\right\}\right]
\end{aligned}
$$

Here $\tau_{w}^{*}$ is the dimensionless shear stress component of the elastico-viscous fluid and the symbols $\mathrm{q}$ and $\mathrm{m}$ represent heat and mass flux and they are given by

$$
q=-k\left(\frac{\partial T^{*}}{\partial y^{*}}\right)_{y^{*}=0} \quad \text { and } \mathrm{m}=-D\left(\frac{\partial C^{*}}{\partial y^{*}}\right)_{y^{*}=0}
$$

\section{RESULTS AND DISCUSSION}

The effects of physical parameters such as magnetic parameter $(M)$, Elastic parameter $\left(\mathrm{K}_{0}\right)$, permeability parameter $\left(k_{p}\right)$, chemical reaction parameter $\left(K_{c}\right)$, heat source parameter $(S)$, Grashoff number for heat transfer $\left(\mathrm{G}_{\mathrm{r}}\right)$, Grashoff number for mass transfer $\left(\mathrm{G}_{\mathrm{c}}\right)$, Prandtl number $\left(\mathrm{P}_{\mathrm{r}}\right)$, Schmidt number $\left(\mathrm{S}_{\mathrm{c}}\right)$ and Phase angle (wt) on the velocity, temperature, concentration profile have been presented with the help of graphs and Tables.

Fig. 1 exhibits the velocity distribution for various values of $G_{r}, G_{c}, K_{0}, k_{p}, K_{c}, S_{c}, \omega$, $\omega t$ and $M$. It is seen that an increase in $G_{r}$ and $G_{c}$ increases the velocity near the plate (curves I, II and IX), due to free convection current which accelerates the velocity. The increase is insignificant in case of thermal buoyancy $\left(\mathrm{G}_{\mathrm{r}}\right)$, whereas it is quite significant in case of mass buoyancy $\left(G_{c}\right)$. Further, it is seen that the presence of transverse magnetic field reduces the velocity due to magnetic interaction, producing Lorentz force which opposes the motion (curves I and X). From the curves XI, XII and I it is observed that the velocity increases in case of generating reaction $\left(K_{c}<0\right)$ but is reversed in case of destructive reaction $\left(K_{c}>0\right)$. It is also observed that the fluid velocity increases due to increase in the elastic parameter $\left(\mathrm{K}_{0}\right)$ and reverse effect is observed in case of phase angle $(\omega t)$ and $S c h m i d t$ number $\left(S_{c}\right)$.

Fig. 2 shows the velocity distribution for various values of $P_{r}$ and $S$. it is seen that higher Prandtl number fluid contributes very insignificantly in increasing the velocity (curves I, III). It is further to note that the heat source parameter reduces the velocity at all points (curve I, II).

Fig.3. depicts the temperature distribution in the flow domain. An increase in frequency parameter $(\omega)$ and heat source parameter (S) contribute to increase the thickness of thermal boundary layer (curves I, IV and VI) but with an increase in the value of Prandtl number $\left(\mathrm{P}_{\mathrm{r}}\right)$ and Phase angle $(\omega t)$ the temperature decreases (curves I, II, III and V). This may be attributed to the fact that smaller values of $\mathrm{P}_{\mathrm{r}}$ lead to increase the thermal conductivity of the fluid and thereby more amount of heat is diffused which causes the decrease in temperature.

Fig. 4 exhibits the concentration distribution for various values of Schmidt number $\left(S_{c}\right)$, chemical reaction parameter $\left(\mathrm{K}_{\mathrm{c}}\right)$, frequency parameter $(\omega)$ and phase angle $(\omega \mathrm{t})$. For heavier species i.e., for higher values of $\left(\mathrm{S}_{\mathrm{c}}\right)$ concentration decreases at all the layers in the flow domain. Similar effects are also observed in case of chemical reaction parameter $\left(\mathrm{K}_{\mathrm{c}}\right)$, frequency parameter $(\omega)$ and phase angle $(\omega \mathrm{t})$.

Table 1 shows that the skin friction $\left(\mathrm{C}_{\mathrm{f}}\right)$ decreases due to higher value of Prandtl number and other parameters such as permeability parameter, magnetic parameter, elastic parameter, chemical reaction parameter, Schmidt number, frequency of oscillation but reverse effect is observed in case of Grashoff numbers, heat source parameter and phase angle $(\omega t)$. 
From Table 2 it is observed that the rate of heat transfer $\left(\mathrm{N}_{\mathrm{u}}\right)$ at the surface increases in case of higher Prandtl number $\left(\mathrm{P}_{\mathrm{r}}\right)$ and frequency of oscillation $(\omega)$ but reverse effect is observed in case of heat source parameter $(\mathrm{S})$ and phase angle $(\omega \mathrm{t})$.

From Table 3 it is observed that the rate of mass transfer $\left(S_{h}\right)$ increases due to increase in Schmidt number, chemical reaction parameters and frequency parameter but decreases with an increase in phase angle.

\section{CONCLUSION}

i) Flow characteristic in the present study is more dependent on mass buoyancy effect $\left(G_{c}\right)$ rather than thermal buoyancy effect $\left(\mathrm{G}_{\mathrm{r}}\right)$.

ii) Magnetic field, chemical reaction parameter and heat source parameter with high Prandtl number flow have a retarding effect on the velocity.

iii) Thinning of thermal boundary layer occurs for higher Prandtl number fluid.

iv) Higher rate of mass transfer is experienced in case of heavier species and in the presence of destructive reaction.

\section{REFERENCES}

[1] V.M. Soundalgekar and P.D. Warne, Unsteady free convection flow past an infinite vertical plate with constant suction and Mass transfer. Journal of Heat and Mass Transfer 20, 1977, 1363-1373.

[2] K.D. Singh, Unsteady free convection flow past a hot vertical porous plate with variable temperature. Proc. Indian Nat. Set. Acad. 58, 1992, 537-544.

[3] K. Anwar, MHD unsteady free convection flow past a vertical porous plate. ZAMM 78, 1998, 255-270.

[4] H. Poonia, and R.C. Chaudhary, MHD free convection and mass transfer flow over an infinite vertical porous plate with viscous dissipation. Theo Appl., Mech., 37, 2010, 263-287.

[5] M. Acharya, , G.C. Dash and L.P. Singh, Magnetic field effects on the free convection and mass transfer flow through porous medium with constant suction and constant heat flux. Indian J. Pure Appl. Maths. 31, 2000, 1-18.

[6] M. Acharya, G.C. Dash and L.P. Singh, Thermal and mass diffusion on unsteady hydromagnetic flow with heat flux and accelerated boundary motion. Indian J. Phys. 76, 2002, 289-296.

[7] K. Walters, Non-Newtonian effects in some elastico-viscous liquids whose behavior at small rates of shear is characterized by a general linear equation of state. Quant. J. Mech. Appl. Math, 15, 1962, 63-76.

[8] P.R. Sharma and D. Pareek, Unsteady flow and heat transfer through an elastico-viscous liquid along an infinite hot vertical porous moving plate with variable free stream suction. Bull. Cal. Math. Sec. 98, 2006, 97-108.

[9] Pravat Kumar Rath, G.C. Dash, and P.K. Rath, Flow and heat transfer of an electrically conducting viscoelastic fluid between two horizontal squeezing/stretching plates. AMSE Modelling Measurement and Control 70, 2001, 45-63.

[10] S.M.B., Alhari, A.A. Mohamed and M. S.E.L. Gerdy, Heat and Mass transfer in MHD viscous elastic fluid flow through a porous medium over a stretching sheet with chemical reaction. Applied Mathematics 1, 2010, 446-455.

[11] B. Kumar and R. Sivaraj, MHD mixed convective visco-elastic fluid flow in a permeable vertical channel with Dufour effect and chemical reaction. Int. J. of Appl. Math. and Mech. 14, 2011, 79-96.

[12] R.A. Damesh and B.A. Shannak, Visco-elastic fluid flow past an infinite vertical porous plates in the presence of first order chemical reaction. Int. J. of Appl. Math. Mech. Engl. Ed. 31, 2010, 955-962.

[13] S. Dash, G.C. Dash and D.P. Mishra, MHD flow through a porous medium past a stretched vertical permeable surface in the presence of heat source/sink and a chemical reaction. Proc. Nat. Acad. Sci. India., 78A, 2008, 49-55.

[14] P.K. Rath, T. Parida and G.C. Dash, Three-Dimension free convective flow through porous medium in a vertical channel with heat source and chemical reaction. Proc. Nat. Acad. Sci. India, 82A, 2012, 225-232.

[15] P.R. Sharma and S. Sharma, Unsteady two dimensional flow and heat transfer through an elasticoviscous liquid along an infinite hot vertical porous surface bounded by porous medium. Bull. Cal. Math. Seoc. 97, 2005; 477-488. 


$$
\begin{aligned}
& \text { APPENDIX } \\
& m_{1}-\left(\frac{P_{r}+\sqrt{P_{r}^{2}-4 P_{r} S}}{2}\right), m_{2}=-\left(\frac{P_{r}+\sqrt{P_{r}^{2}-4 P_{r}\left(S-\frac{i \omega}{4}\right)}}{2}\right) \\
& m_{3}=-\left(\frac{S_{c}+\sqrt{S_{c}^{2}+4 K_{c} S_{c}}}{2}\right), \quad \alpha_{1}=-\left(\frac{i w}{4}+K_{c}\right) S_{c} \text {, } \\
& m_{4}=-\left(\frac{S_{c}+\sqrt{S_{c}^{2}+4 \alpha_{1}}}{2}\right), \alpha_{2}=M+\frac{1}{k_{p}}, \quad m_{5}=-\left(\frac{1+\sqrt{1+4 \alpha_{2}}}{2}\right), m_{6}=-\left(\frac{1+\sqrt{1+4 \alpha_{2}+\frac{i \omega}{4}}}{2}\right) \\
& \mathrm{A}_{1}=m_{1}^{2}+m_{1}-\alpha_{2}, \quad \mathrm{~A}_{2}=m_{3}^{2}+m_{3}-\alpha_{2}, \quad \mathrm{~A}_{3}=\frac{G_{r}}{A_{1}}, \quad \mathrm{~A}_{4}=\frac{G_{c}}{A_{2}}, \quad \mathrm{~A}_{5}=m_{5}^{2}+m_{5}-\alpha_{2} \\
& \mathrm{~A}_{6}=\frac{\left(1-A_{3}-A_{4}\right) m_{5}^{3}}{A_{1}}, \quad \mathrm{~A}_{7}=\frac{A_{3} m_{1}^{3}}{A_{1}}, \quad \mathrm{~A}_{8}=\frac{A_{4} m_{3}^{3}}{A_{2}} \\
& \mathrm{~A}_{9}=-\left(\mathrm{A}_{7}+\mathrm{A}_{8}\right), \quad \mathrm{A}_{10}=\frac{-G_{r}\left(1+\frac{4 i}{\omega} m_{1}\right)}{m_{2}^{2}+m_{2}-\left(\alpha_{2}+\frac{i \omega}{4}\right)}, \quad \mathrm{A}_{11}=\frac{G_{r} \frac{4 i}{\omega} m_{1}}{A_{1}-\frac{i \omega}{4}} \\
& \mathrm{~A}_{12}=\frac{-G_{c}\left(1+\frac{4 i}{\omega} m_{3}\right)}{m_{4}^{2}+m_{4}-\left(\alpha_{2}+\frac{i \omega}{4}\right)}, \mathrm{A}_{13}=\frac{G_{c} \frac{4 i}{\omega} m_{3}}{A_{2}-\frac{i \omega}{4}}, \mathrm{~A}_{14}=-\frac{\left(A_{3}+A_{4}-1\right) m_{5}}{A_{5}-\frac{i \omega}{4}} \\
& \mathrm{~A}_{15}=\frac{A_{3} m_{1}}{A_{1}-\frac{i \omega}{4}}, \mathrm{~A}_{16}=\frac{A_{4} m_{3}}{A_{2}-\frac{i \omega}{4}}, \\
& \mathrm{~A}_{17}=\mathrm{A}_{11}+\mathrm{A}_{15}, \mathrm{~A}_{18}=\mathrm{A}_{13}+\mathrm{A}_{16}, \mathrm{~A}_{19}=\left(-1-\mathrm{A}_{17}-\mathrm{A}_{18}-\mathrm{A}_{10}-\mathrm{A}_{14}-\mathrm{A}_{12}\right) \\
& \mathrm{A}_{20}=\mathrm{m}_{6}^{2}+m_{6}-\alpha_{2}, \quad \mathrm{~A}_{21}=\mathrm{m}_{2}^{2}+m_{2}-\alpha_{2}, \mathrm{~A}_{22}=\mathrm{m}_{4}^{2}+m_{4}-\alpha_{2} \\
& \mathrm{~A}_{23}=\frac{\left(\frac{\mathrm{i} \omega}{4} \mathrm{~m}_{6}^{2}-m_{6}^{3}\right) A_{19}}{A_{20}-\frac{\mathrm{i} \omega}{4}}, \mathrm{~A}_{24}=\frac{\left(\frac{\mathrm{i} \omega}{4} \mathrm{~m}_{1}^{2}-m_{1}^{3}\right) A_{17}}{A_{1}-\frac{\mathrm{i} \omega}{4}} \\
& \mathrm{~A}_{25}=\frac{\left(\frac{\mathrm{i} \omega}{4} \mathrm{~m}_{3}^{2}-m_{3}^{3}\right) A_{18}}{A_{2}-\frac{\mathrm{i} \omega}{4}}, \mathrm{~A}_{26}=\frac{\left(\frac{\mathrm{i} \omega}{4} \mathrm{~m}_{2}^{2}-m_{2}^{3}\right) A_{10}}{A_{21}-\frac{\mathrm{i} \omega}{4}},
\end{aligned}
$$


$\mathrm{A}_{27}=\frac{\left(\frac{\mathrm{i} \omega}{4} \mathrm{~m}_{5}^{2}-m_{5}^{3}\right) A_{14}}{A_{5}-\frac{\mathrm{i} \omega}{4}}, \mathrm{~A}_{28}=\frac{\left(\frac{\mathrm{i} \omega}{4} \mathrm{~m}_{4}^{2}-m_{4}^{3}\right) A_{12}}{A_{22}-\frac{\mathrm{i} \omega}{4}}, \quad \mathrm{~A}_{29}=\frac{-\left(\mathrm{A}_{3}+A_{4}-1\right) m_{5}^{3}}{A_{5}-\frac{\mathrm{i} \omega}{4}}, \mathrm{~A}_{30}=\frac{\mathrm{A}_{3} m_{1}^{3}}{A_{1}-\frac{\mathrm{i} \omega}{4}}$, $\mathrm{A}_{31}=\frac{A_{4} m_{3}^{3}}{A_{2}-\frac{\mathrm{i} \omega}{4}}, \mathrm{~A}_{32}=\frac{-A_{9} m_{5}}{A_{5}-\frac{\mathrm{i} \omega}{4}}, \mathrm{~A}_{33}=-\frac{A_{7} m_{1}}{A_{1}-\frac{\mathrm{i} \omega}{4}}, \mathrm{~A}_{34}=-\frac{A_{8} m_{3}}{A_{2}-\frac{\mathrm{i} \omega}{4}}$

$A_{35}=A_{27}+A_{29}+A_{32}, A_{36}=A_{25}+A_{31}+A_{34}, A_{37}=A_{22}+A_{30}+A_{33}, A_{38}=-\left(A_{35}+A_{28}+A_{36}+A_{26}+A_{37}\right)$

TABLE - 1 Skin friction $\left(C_{f}\right)$

\begin{tabular}{|c|c|c|c|c|c|c|c|c|c|c|r|}
\hline $\boldsymbol{G}_{\boldsymbol{r}}$ & $\boldsymbol{P}_{\boldsymbol{r}}$ & $\boldsymbol{K}_{\boldsymbol{p}}$ & $\boldsymbol{\omega}$ & $\boldsymbol{\omega} \boldsymbol{t}$ & $\boldsymbol{K}_{\boldsymbol{o}}$ & $\boldsymbol{G}_{\boldsymbol{c}}$ & $\boldsymbol{M}$ & $\boldsymbol{K}_{\boldsymbol{c}}$ & $\boldsymbol{S}_{\boldsymbol{c}}$ & $\boldsymbol{S}$ & $\boldsymbol{C}_{\boldsymbol{f}}$ \\
\hline 5 & 5 & 0.4 & 5 & $\pi / 4$ & 0.2 & 2 & 2 & 1 & 0.22 & 1 & 5.906 \\
\hline 7 & 5 & 0.4 & 5 & $\pi / 4$ & 0.2 & 2 & 2 & 1 & 0.22 & 1 & 6.236 \\
\hline-5 & 5 & 0.4 & 5 & $\pi / 4$ & 0.2 & 2 & 2 & 1 & 0.22 & 1 & 4.249 \\
\hline-7 & 5 & 0.4 & 5 & $\pi / 4$ & 0.2 & 2 & 2 & 1 & 0.22 & 1 & 3.918 \\
\hline 5 & 7 & 0.4 & 5 & $\pi / 4$ & 0.2 & 2 & 2 & 1 & 0.22 & 1 & -442.203 \\
\hline 5 & 5 & 100 & 5 & $\pi / 4$ & 0.2 & 2 & 2 & 1 & 0.22 & 1 & -1.971 \\
\hline 5 & 5 & 0.4 & 15 & $\pi / 4$ & 0.2 & 2 & 2 & 1 & 0.22 & 1 & -1.328 \\
\hline 5 & 5 & 0.4 & 5 & $\pi / 3$ & 0.2 & 2 & 2 & 1 & 0.22 & 1 & 11.626 \\
\hline 5 & 5 & 0.4 & 5 & $\pi / 4$ & 0 & 2 & 2 & 1 & 0.22 & 1 & 22.009 \\
\hline 5 & 5 & 0.4 & 5 & $\pi / 4$ & 0.2 & 5 & 2 & 1 & 0.22 & 1 & 10.885 \\
\hline 5 & 5 & 0.4 & 5 & $\pi / 4$ & 0.2 & 2 & 5 & 1 & 0.22 & 1 & 2.269 \\
\hline 5 & 5 & 0.4 & 5 & $\pi / 4$ & 0.2 & 2 & 2 & -0.04 & 0.22 & 1 & 9.202 \\
\hline 5 & 5 & 0.4 & 5 & $\pi / 4$ & 0.2 & 2 & 2 & 0 & 0.22 & 1 & 8.780 \\
\hline 5 & 5 & 0.4 & 5 & $\pi / 4$ & 0.2 & 2 & 2 & 1 & 0.78 & 1 & 2.577 \\
\hline 5 & 5 & 0.4 & 5 & $\pi / 4$ & 0.2 & 2 & 2 & 1 & 0.22 & 0.5 & -76.803 \\
\hline
\end{tabular}

TABLE - 2 Nusselt number $\left(N_{u}\right)$

\begin{tabular}{|c|c|c|c|r|}
\hline $\boldsymbol{P}_{\boldsymbol{r}}$ & $\boldsymbol{\omega}$ & $\boldsymbol{\omega t}$ & $\boldsymbol{S}$ & \multicolumn{1}{c|}{$\boldsymbol{N}_{\boldsymbol{u}}$} \\
\hline 5 & 5 & $\pi / 4$ & 1 & 6.04581 \\
\hline 7 & 5 & $\pi / 4$ & 1 & 8.81121 \\
\hline 11.4 & 5 & $\pi / 4$ & 1 & 6.68664 \\
\hline 5 & 15 & $\pi / 4$ & 1 & 5.77632 \\
\hline 5 & 5 & $\pi / 3$ & 1 & 7.20342 \\
\hline 5 & 5 & $\pi / 4$ & 0 & 8.16158 \\
\hline 5 & 5 & $\pi / 4$ & -1 & \\
\hline
\end{tabular}

TABLE - 3 Sherwood number $\left(S_{h}\right)$

\begin{tabular}{|c|c|c|c|r|}
\hline $\boldsymbol{S}_{\boldsymbol{c}}$ & $\boldsymbol{\omega}$ & $\boldsymbol{\omega t}$ & $\boldsymbol{K}_{\boldsymbol{c}}$ & $\boldsymbol{S}_{\boldsymbol{h}}$ \\
\hline 1.002 & 5 & $\pi / 4$ & 1 & 1.69103 \\
\hline 0.600 & 5 & $\pi / 4$ & 1 & 1.17297 \\
\hline 0.780 & 5 & $\pi / 4$ & 1 & 1.41045 \\
\hline 1.002 & 5 & $\pi / 4$ & 0 & 1.35615 \\
\hline 1.002 & 5 & $\pi / 4$ & 1.5 & 1.75429 \\
\hline 1.002 & 5 & $\pi / 4$ & 2 & 1.78982 \\
\hline 1.002 & 15 & $\pi / 4$ & 1 & 1.88002 \\
\hline 1.002 & 5 & $\pi / 3$ & 1 & 1.52351 \\
\hline
\end{tabular}




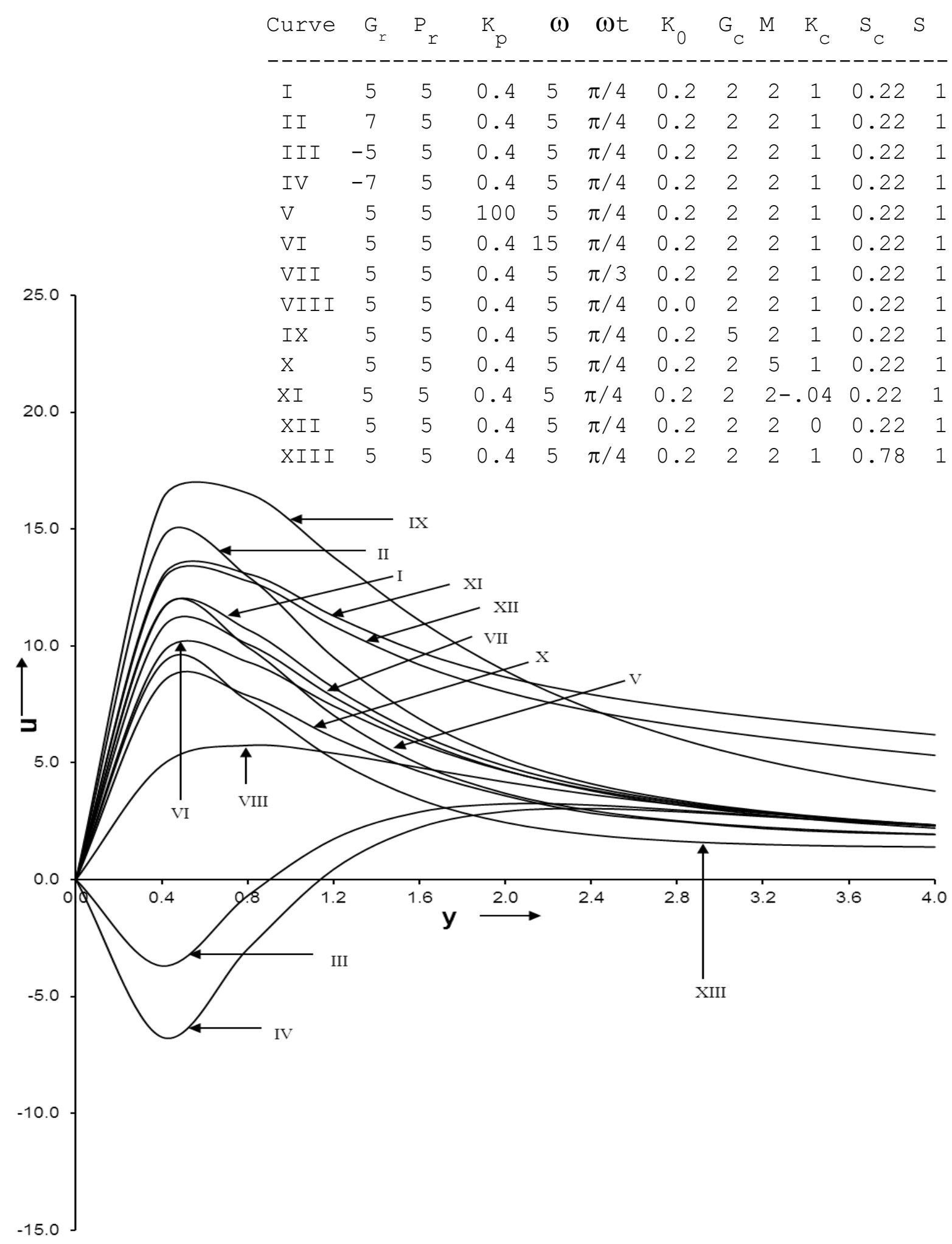

Fig- 1 Velocity distribution for various values of $G_{r}, k_{p}, \omega, \omega t, K_{0}, G_{c}, M, S_{c}$ and $K_{c}$ when $\varepsilon$ $=0.5, P_{r}=5$ and $S=1$ 


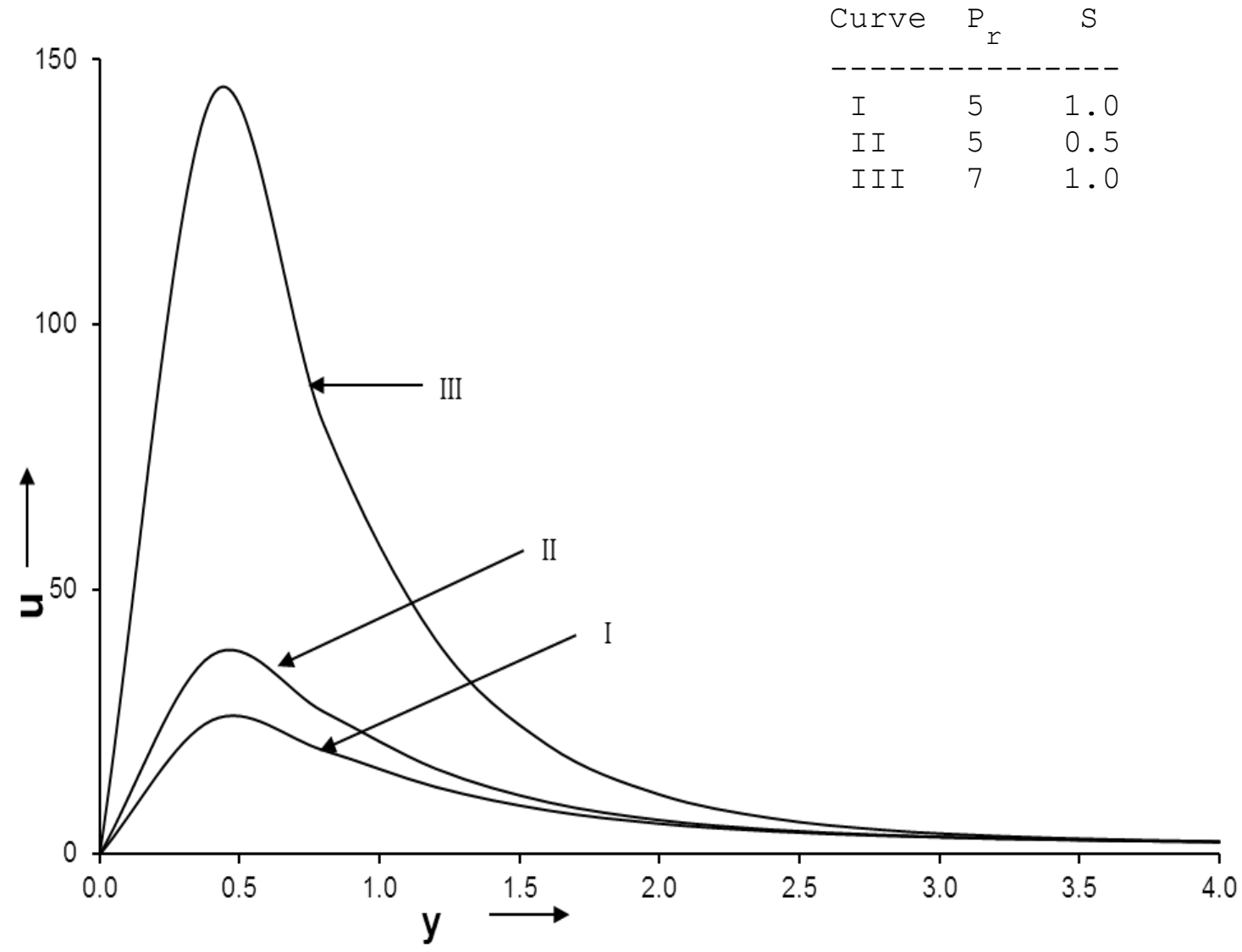

Fig- 2 Velocity distribution for various values of $P_{r}$ and $S$ when $\varepsilon=0.5, G_{r}=5$, $\omega \mathrm{t}=\pi / 4, \mathrm{~K}_{\mathrm{p}}=0.4, \omega=5, \mathrm{~K}_{0}=0.2, \mathrm{G}_{\mathrm{c}}=2, \mathrm{M}=2, \mathrm{~K}_{\mathrm{c}}=1 \& \mathrm{~S}_{\mathrm{c}}=0.22$. 


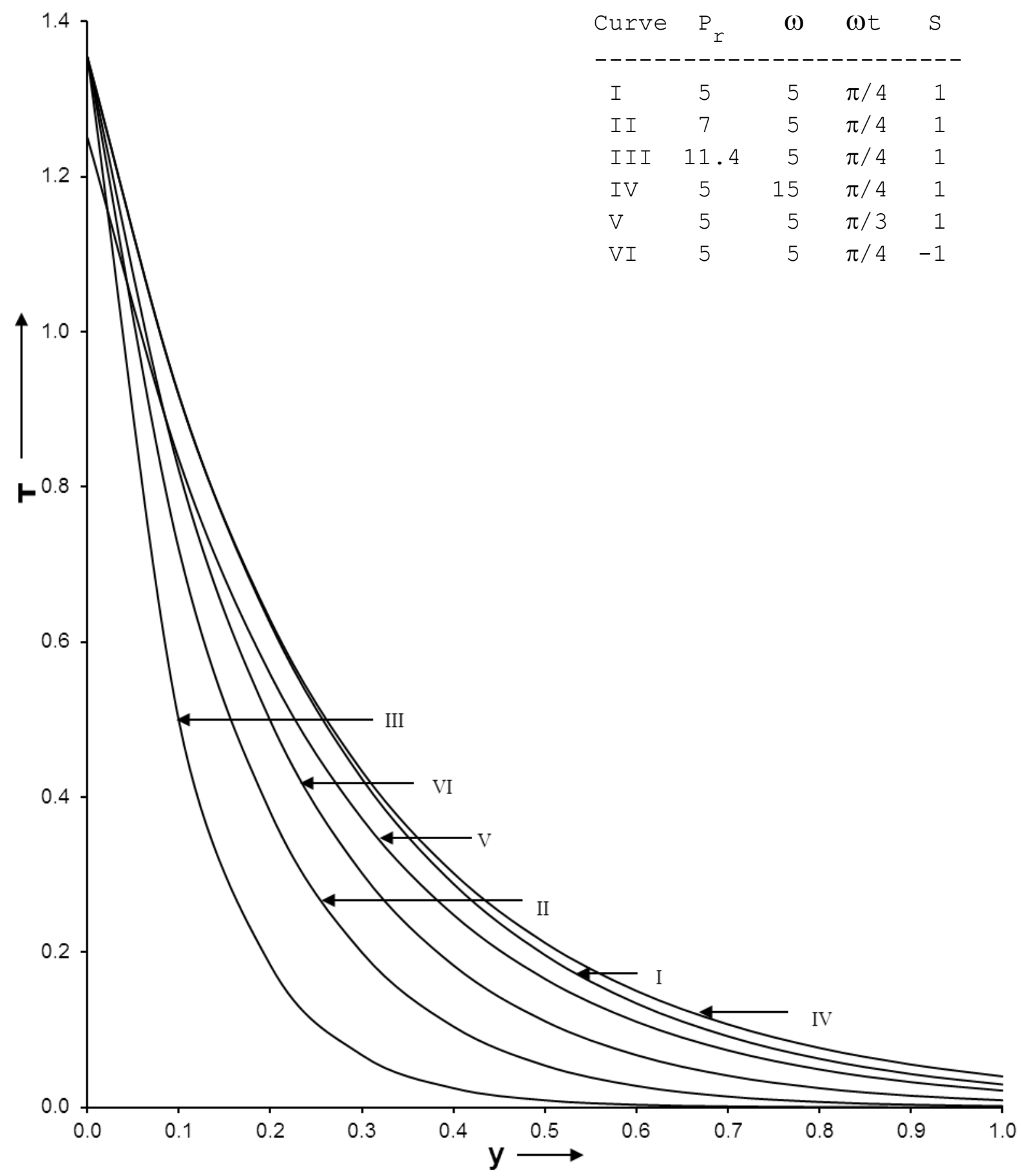

Fig- 3 Temperature distribution for $\varepsilon=0.5$ 


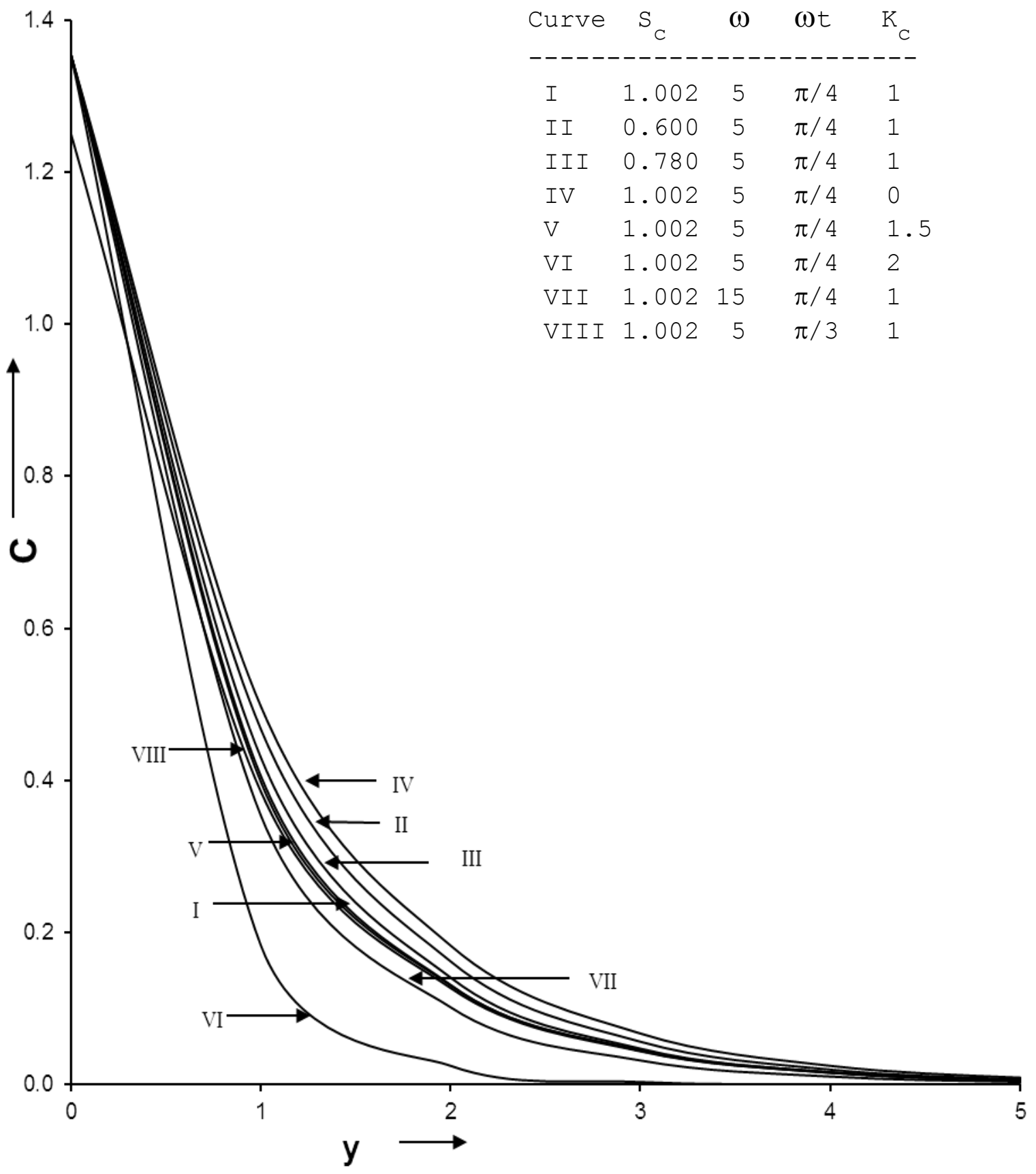

Fig- 4 Concentration distribution for $\varepsilon=0.5$ 